\title{
Combined Resource Allocation System for Device-to-Device Communication towards LTE Networks
}

\author{
Fakhar Abbas ${ }^{1}$, Ye Fang ${ }^{1}$, Muhammad Irshad Zahoor ${ }^{1}$, and Kashif Sultan ${ }^{2}$ \\ ${ }^{1}$ College of information and Communication Engineering, Harbin Engineering University, Harbin, PR, China. \\ ${ }^{2}$ School of Computer and Communication Engineering, University of Science and Technology Beijing, Beijing, PR, China.
}

\begin{abstract}
The LTE networks are being developed to grant mobile broadband services in the fourth generation (4G) systems and allow operators to use spectrum more efficiently.D2D communication is a promising technique to provide wireless services and enhance spectrum exploitation in the LTE Heterogeneous Networks (HetNets).D2D communication in HetNets allows users to communicate with each other directly by reusing the resources when communicating via the base stations. But during the downlink period, both the D2D receiver and the Heterogeneous Users equipment's (HUE) experience interference caused by resource allocation. In this article, we identify and analyze the interference problem of HetNets caused by D2D transmitter during download. We propose a combined resource allocation and resource reuse method for LTE HetNets, where resource allocation to HUEs is employed on the basis of comparative fair algorithm and resource reuse to D2D users is employed on acquisitive empirical algorithm. This approach evaluates whether D2D mode is suitable or not by path loss evaluation, after that decreases the interference to HUE by selection of the minimum channel gain between HUE and D2D transmitter each time to mitigate interference. Our simulation results show that the efficiency and throughput of HetNets is improved by using the proposed method.
\end{abstract}

\section{Introduction}

As demands for high data rates increasing, the deficiency of radio resources impedes further growth of HetNets services. D2D communication tends to solve the problem by improving spectrum efficiency significantly. D2D communication enables mobile devices to communicate directly with each other, through reusing resources in HetNets [1] [2]. D2D communication scheme is incorporated which brings benefits in three ways. Firstly, D2D communication can increase the system throughput and the spectral efficiency by reusing the user resource. Secondly, the D2D communication decreases the transmission power of D2D transmitter. Thirdly, D2D communication has the advantage that licensed spectrum is allocated to local communication thus ensuring a secure and robust (against interference) environment [2]. D2D communication is also increasing necessity for local communication, such as games between mobile devices, video sharing, data flooding, local advertisement and information broadcast in hot point area like market. Machine to Machine communications in small distance in the Internet things also becomes a focus, as a local communication technology [2].
A major challenge in D2D communication is interference between different devices. Several attempts have been made to deal with the interference issues in D2D communication for LTE Networks. The D2D communication described in [3] can increase system throughput by reusing the resource of the cellular user. The interference cellular communication and D2D communication can be coordinated through proper power control and resource allocation. The D2D communication reduces the transmit power of terminal, which increases the working time of terminal and improves the energy efficiency. D2D communication can also decrease the load of base station (BS) via direct transportation. Besides, the D2D communication has the advantage that licensed spectrum is allocated to local communication. Licensed spectrum can guarantee a planned situation instead of an uncoordinated one. In [2], the method for D2D communication session association and organization involving procedures in the LTE System Architecture Evolution are proposed. The BS is capable of coordinating the interference between cellular communication and D2D communication by proper power control and resource allocation with the channel state information (CSI) of all involved links. In [4], a resource allocation method has been proposed to reduce the system interference. It, however, consisted of limited number of HUE and D2D pairs, and one D2D pair reused 
the resource of only one HUE in the system model. In [5] authors described three D2D resource allocation methods and the power optimization for only one cellular user and one D2D pair. In [6] the authors described mode selection method on the basis of approached presented in [5]. The authors in [7] proposed two methods to coordinate the mutual interference between cellular communication and D2D systems. The authors in [8] proposed time hopping (TH) based radio resource allocation schemes to improve the robustness of the hybrid network. These above mentioned approaches are based on the use of stand-alone schemes. This is, however, an interesting task to investigate the use of combined schemes.

Our aim is to reduce the interference which caused the interference to primary service in non-orthogonal method. The BS coordinates the transmit power for both links. We focus on the case in which HUE and D2D pairs are multiplexed on the same downlink OFDM physical resource block (PRB). Time Division Duplex (TDD) is used for the proposed scheme. Two UEs in a D2D pair share the given PRBs by time. Taking inspiration from the approach presented in [9] [10], this approach further seeks to improve resource allocation. First, there is a subcarrier and power combine allocation scheme to HUEs. We calculate the simulation result on both the comparative fair $(\mathrm{CF})$ and Round Robin (RR) algorithm. Then, subcarrier reuse allocation scheme to the D2D pairs. We also present the comparison of path loss and the Signal to interference and noise ratio (SINR) to the threshold. Path loss is the main element used for approach selection. If the path loss of two D2D UEs is smaller than between any of them to the BS, the D2D mode is preferred. The resource reuse allocation is activated if the SINR of receiver in both of the HUE and the D2D pair is equal or bigger than the minimum allowed SINR.

The rest of the paper is organized as follows: In Section II, we present the system model. We formulate the problem in Section III. We then discuss the proposed approach in Section IV. Resource allocation and reuse algorithm are discussed in Section V. We present the simulations results in Section VI. Finally, we conclude the paper in Section VII.

\section{System Model}

In this part, we described network model and channel model with theoretical and mathematical analysis.

\subsection{Network Model}

In LTE system, the bandwidth is divided into equal size physical resource blocks (RBs). Each physical resource block (PRB) exists in 0.5 milli seconds time slots corresponding to $180 \mathrm{kHz}$ in the frequency domain with subcarrier spacing of $15 \mathrm{kHz}$. In LTE, the multiple access method for downlink (DL) is Orthogonal Frequency Division Multiple access (OFDMA).Fig.1 shows the high level overview of LTE DL physical resource architecture.
LTE adopts Orthogonal Frequency Division Multiplex (OFDM) based radio interface due to its higher spectral efficiency and flexibility against multi path delay spread. The physical properties of SC-FDMA require that RBs allocated to a single user to be contiguous in frequency. The minimum scheduling period in the frequency domain is one physical RB, therefore the smallest unit of resource that can be assigned comprises of two RBs. In this architecture, D2D UEs may communicate directly over the D2D links. However, BS needs to establish the D2D connection and also remains in control of resource allocation to limit the interference experienced by the HetNets receivers. The SINR is calculated from received signal and interference power level.

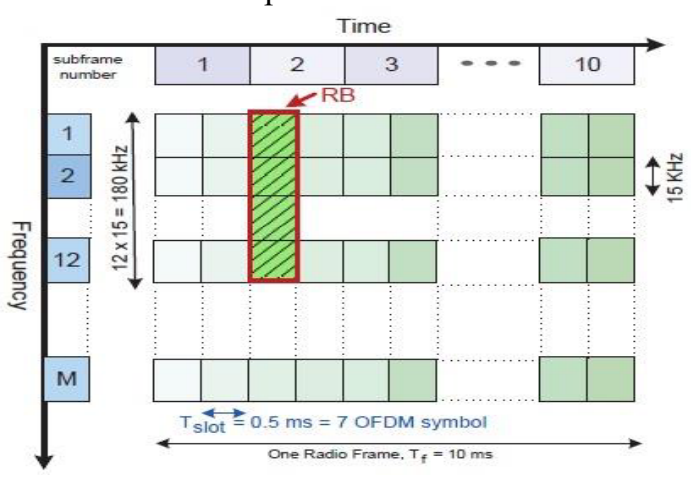

Figure 1. LTE Downlink physical resource

In order to guarantee the reuse of the HetNets resource, the interférence must be controlled well in a required range as provided by (1) and (2).

$$
\begin{aligned}
& \operatorname{SINR}_{H U E}<=S I N R_{\text {threshold }} \\
& \operatorname{SINR}_{d 2 d\left(U E_{1}\right)}<=S I N R_{\text {thershold }}
\end{aligned}
$$

Equation (1) describes signal to interference and noise ratio (SINR) of Heterogeneous Users equipment's (HUE) which is less than the threshold level. "(2)," describes Signal to interference and noise ratio (SINR) of D2D user (UE1) is less the threshold level. The interference depends on the transmitter power and the distance between the two devices. Consequently intelligent selection of the physical resource block (PRB) for reuse would result in improved performance in terms of network throughput. If D2D UE's pair is close to the BS and the path loss between any of them and the BS is bigger than the path loss of the D2D pair, the HUE will suffer from significant interference which is troublemaking to the network throughput. To make sure that the D2D mode is ideal; the path loss of two D2D UEs must satisfy the following condition.

\subsection{Channel Model}

To evaluate important parameters SINR and channel gain, we consider both distant dependent path loss and shadow fading path loss. $f_{c}$ Is the carrier frequency, $\mathrm{d}[\mathrm{m}]$ is distance between base station and user while $h_{B S}$ is the BSs antenna height. BS device path loss parameter for 
Line-of-sight (LOS) and Non-Line-sight (NLOS) is as follows:

$$
\begin{aligned}
& \text { Pathloss }=22 \cdot \log 10\left(d[m]+42.0+20 \log 10\left(f_{c}[G h z] / 5\right)\right. \\
& \text { Pathloss }=36.5 \cdot \log 10\left(d[m]+40.9+26 \log 10\left(f_{c}[G h z] / 5\right)\right.
\end{aligned}
$$

D2D communication path loss parameters for LOS and NLOS are as follows:

$$
\begin{aligned}
& \text { Pathloss }=15.9 \cdot \log 10\left(d[m]+46.7+20 \log 10\left(f_{c}[G h z] / 5\right)\right. \\
& \text { Pathloss }=40 . \log 10\left(\mathrm{R}[\mathrm{km}]+30 \log 10\left(f_{c}[M h z] / 5\right)+49\right.
\end{aligned}
$$

$\mathrm{R}$ is the $\mathrm{D} 2 \mathrm{D}$ separation, $f_{c}$ is carrier frequency. The slow fading or shadowing on a wireless channel is caused by obstacles in the propagation path of the radio waves and is location dependent [11]. When shadowing is modeled only by $\log$ normally distributed random variables, the variables do not meet the spatial correlation properties. Finding an accurate fading model is a parable the slow fading or shadowing on a wireless channel is caused by obstacles in the propagation path of the radio waveband obviously is location dependent. The linear power gain between the BS and a user is as follows

$$
\text { Gain }=10^{-(P L+s h d) / 10}
$$

Power gain between two users for D2D communication is as follows.

$$
\text { Gain }=10^{-(\mathrm{PL}) / 10}
$$

\section{Problem Definitions}

D2D commutation takes place in the primary HetNets. We identify the radio resources to be important and D2D communication shares the same resources with HetNets communication simultaneously rather than using dedicated resources. Therefore, the interference of D2D communications to the HetNets needs to be restricted to maintain a target performance level of the HetNets. If the distance between $\mathrm{D} 2 \mathrm{D}$ is too long, the $\mathrm{D} 2 \mathrm{D}$ will require high power. In that case, the primary HetNets would experience from interference.

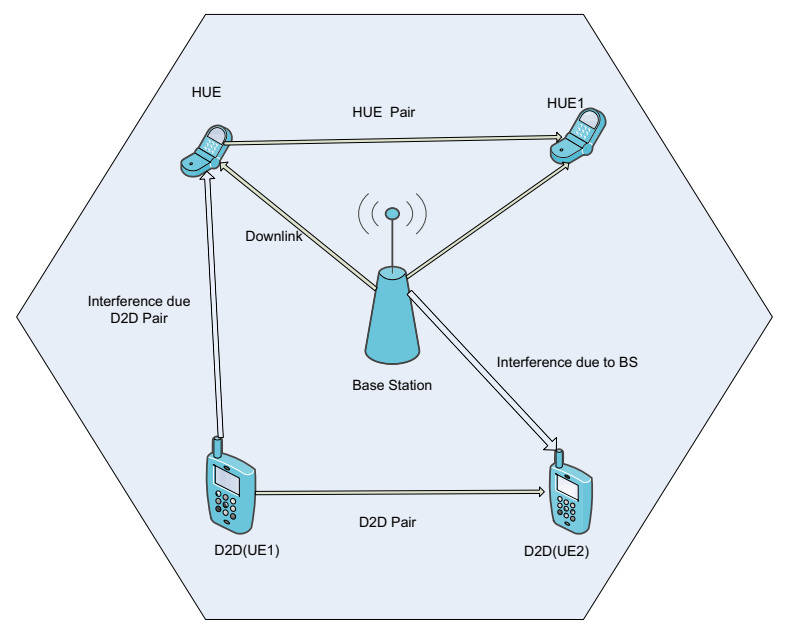

Figure 2. Interference problem in LTE Downlink when HetNets shares channel with the D2D network.
Fig.2 illustrates the interference problem when primary HetNets service shares channel with the D2D service in LTE Downlink network with HUE and D2D pair (D2D(UE1)-transmitter, D2D(UE2) -receiver).

During the downlink of HetNets, HUE is exposed to interference when D2D (UE1) transmits using the same allocated sub-band. Also, D2D receiver UE2 will suffer interference from the base station (BS), whichever shares the same sub-band with the HetNets service. It can be estimated that the amount of interference does not depend only on D2D transmit power but also on spatial distance between D2D transmitter and the HUE. According to analysis of power control scheme for D2D communication, we reject $\mathrm{D} 2 \mathrm{D}$ connections if the maximum distance between D2D is higher. Further we assume that both UEs need to be in the same network for possible connection.

\section{Proposed Approach}

The base stations (BS) schedules the primary HetNets in normal mode, and we allocate one resource block from the RBs assigned to HUE to D2D link. The total number of available RBs for the Downlink is M. The BS serves a set $\mathrm{H}$ of HUEs and a set D of D2D pairs. We formulate the problem of assigning appropriate RBs for underlying D2D communication as an optimization problem that achieves higher throughput without impairing the existing HetNets. The underlying assumption is $H>D$.

\subsection{UE Resource allocation}

For primary HUE service, to optimize bandwidth allocation in the system such that the data rate requirements of a maximum number of users can be fulfilled. Aim is to find a bandwidth allocation that allows each user to satisfy its rate condition while maximizing the sum of user data rates subject to total power limit. The rate adaptive (RA) optimization problem can be state as below:

$$
\max _{x_{h m}, p_{h m}} \operatorname{imize}_{h=1}^{H} \sum_{m=1}^{M} x_{h m} b_{h m}
$$

Where $\mathrm{H}$ is the set of HUE and $\mathrm{M}$ is the total number of available resource block, $h$ is the number of HUE, $x_{h m} \in\{0,1\}$ is subcarrier allocation indicator for all values of HUE, m. $p_{h m}>=0$ is power for all value of $\mathrm{h}$, m. And $\sum_{h=1}^{H} \sum_{m=1}^{M} p_{h m} \leq P_{T}$, where $P_{T}$ is the overall transmitting power constraint. $\sum_{h=1}^{H} x_{h m}<=1$, for all values of $m \in\{1,2,3,4, \ldots ., \mathrm{M}\}, R_{i}: R_{j}=\phi_{i}: \phi_{i}$ overall power and user proportionality constraints for all values of $i, j \in\{1,2,3, \ldots . ., H\}$. Normalized transmit rate is $R_{h} / \phi_{h}$, where $\phi_{h}$ is rate influence of HUE. The 
throughput $b_{h m}$ is determined by the Shannon capacity model in equation (8). $p_{h m}$ Is received power, $N_{0}$ is the receiver's noise, $I$ inter-cell interference and $G_{b h}$ is the channel power gain between the base station (BS) and HetNets $U E_{h}$.

$$
b_{h m}=\log 2\left(1+\frac{p_{h m} G_{b h}}{N_{0}+1}\right)
$$

For user $h$, the total granted transmit throughput can be calculated as fallows, where B is the total bandwidth.

$$
R_{h}=\frac{B}{M} \sum_{m=1}^{M} x_{h m} b_{h m}
$$

During the download phase of HetNets, any UE is exposed to interference when any D2D user is allowed to transmit using the same allocated sub band. The amount of interference will depend not only on D2D transmit power but also on the channel gain between D2D transmitter and the HUE.

\subsection{UE Resource allocation}

The downlink signal to interference noise ratio of user Het (UE) is as follows:

$$
S I N R_{h}=\frac{P_{B} G_{B h}}{N_{0}+I+\sum_{m=1}^{M} x_{d h} P_{d 2 d\left(U E_{1}\right)} G_{d 2 d\left(\mathrm{UE}_{1}\right) h}}
$$

The signal to interference noise ratio for the device to device pair receiver $d 2 d\left(U E_{1}\right)$ is as follows:

$$
S I N R_{d 2 d\left(\mathrm{UE}_{2}\right)}=\frac{P_{d 2 d\left(U E_{1}\right)} G_{d 2 d\left(U E_{1}, \mathrm{UE}_{2}\right)}}{N_{0}+I+\sum_{m=1}^{M} x_{d h} P_{B} G_{B d 2 d\left(\mathrm{UE}_{2}\right)}}
$$

$G_{B d 2 d(\mathrm{UE} 2)}$ Is the channel gain between BS and d2d, $P_{d 2 d\left(U E_{1}\right)}$ is D2D user received power. $G_{d 2 d\left(U E_{1}, U E_{2}\right)}$ Denotes the channel gain between two D2D UEs, $P_{B}$ is BS power, $N_{0}$ is receiver's noise and thermal noise density and I is inter-cell interference.

For resource reuse method, we need to maximize the sum rates of the primary HUE and secondary D2D UEs by applying the Shannon capacity formula. To maximize the sum throughput of D2D and HUE when sharing DL resources of HUE, the problem is formulated as follow:

$$
\max _{x_{d m}, p_{d m}} \text { imize } \sum_{d=1}^{D} \sum_{m=1}^{M} x_{d m} b_{d m}+\sum_{h=1}^{H} \sum_{d=1}^{D} \sum_{m=1}^{M} x_{h m} x_{d m}\left(b_{h m}^{c}-b_{h m}\right) \text { (12) }
$$

Where $x_{d m} \in\{0,1\}$ for all values of $d, m$ and $p_{d m} \geq 0$ is power for all values of $d, m$. D is total number of D2D pairs; $M$ is total number of available resource blocks during the downlink and $\mathrm{H}$ is the set of HUEs that BS serves. $\sum_{d=1}^{D} x_{d m}<=1$ For all values of $m \in\{1,2,3, \ldots . ., \mathrm{M}),\left(b_{h m}^{c}-b_{h m}\right)$ is the throughput difference of HUE.

$$
\begin{aligned}
& S I N R_{H U E}<=S I N R_{\text {threshold }} \\
& S I N R_{d 2 d\left(U E_{1}\right)}<=S I N R_{\text {thershold }}
\end{aligned}
$$

Signal to interférence noise (SINR) condition to control interference. The amount of interference depends on transmitted power and distance between two users.

$$
\begin{aligned}
& P L_{D 2 D}<=P L_{d 2 d\left(U E_{1}\right), B S} \\
& P L_{D 2 D}<=P L_{B S, d 2 d\left(U E_{2}\right)}
\end{aligned}
$$

The path loss of two D2D UEs must satisfy the above conditions. The speed of $b_{h m}$ is determined by Shannon capacity formula with inter-cell interference. The throughput of $b_{h m}$ is similar to $b_{d m}$.

$$
b_{h m}=\log 2\left(1+S I N R_{h}\right)
$$

The sum throughput of whole scheme is the sum of two equations (7) and (12).

\section{Combined resource allocation and resource reuse method}

The allocation of resources between D2D and HetNets is determined by the Base station. If D2D users are assigned resources that are orthogonal to those occupied by the HUE, they cause no interference to each other and the analysis is simpler. On the other hand, the resource usage efficiency can be higher in nonorthogonal mode.

This work aims at reducing the interference in the non-orthogonal mode. Under non-orthogonal mode, the D2D and HUE reuse the same resources, causing interference to each other. The BS coordinates the transmit power for both links. The most constructive solution to the problem of rate adaptive optimization of equation (7) should iteratively combine both the subcarrier allocation and bit allocation. In the bit allocation step, the total power is assumed to be allocated to subcarriers according to the same SINR. According to $R_{i}: R_{j}=\phi_{i}: \phi_{i}$ for all values of $i, j \in\{1,2,3, \ldots . ., H\}$, the subcarrier allocation method initially determines the number of subcarriers allocated to each user. In order to assure user rate proportionality, the user with the minimum normalized transmit rate $R_{h} / \phi_{h}$ is permitted to choose a subcarrier at each iteration.

Below is the summarized proposed proportional subcarrier allocation algorithm. This is an Acquisitive policy, where the user with the minimum stabilize transmit rate is preferred to choose a best subcarrier each time. 


\section{Algorithm 1: HetNets Service RBs Distribution method \\ 1. Initialization. \\ 2. For $H U E=1: H$, Sorted list of $b_{h m}$ in ascending order, HUE is a user to find the optimal physical resource blocks $m *=\arg \max _{m \in s} b_{h m}$}

3. Set $x_{h m^{*}}=1$, Removal of resources allocated from the set of subcarriers $S=S-\{\mathrm{m} *\}$.

4. if $S \neq \varnothing$, determine the set of subcarriers is empty, then

5. Find out each iteration normalized transmission bit rate of the minimal user $h=\arg \min _{h}\left\{R_{h} / \phi_{h}\right\}$.

6. Find the optimal allocation of resource blocks to the user $m^{*}=\arg \max _{m \in S} b_{h * m *}$

7. Set $x_{h * m *}=1, S=S-\{\mathrm{m} *\}$ until $S=\varnothing$.

Below is the D2D service reuse resource block distribution method. This is an alternative acquisitive empirical RBs selection algorithm. From Equation

$\max \operatorname{imize}_{x_{h m}, p_{h k}} \sum_{h=1}^{H} \sum_{m=1}^{M} x_{h m} b_{h m}$, we find That lower channel gain will result in higher $\operatorname{SINR}_{h}$. Therefore, we select primary UE with higher channel quality indicator split $\mathrm{RB}$ with the $\mathrm{D} 2 \mathrm{D}$ pair with lower channel gain.

\section{Algorithm 2: D2D Service RBs Distribution method}

1. Initialization.

2. Set $H U E=1: H, d=1: \mathrm{D}$ while $D \neq \varnothing$ and $H U E \leq H$, HUE to select the optimal or suboptimal reusable resources $h *=\arg \max \operatorname{sum}\left(x_{h m}\right)$, the pairing of D2D users $d *=\arg \min G_{d 2 d\left(U E_{1}\right) \mathrm{h} *}$

3. If $P L_{D 2 D}<=P L_{d 2 d\left(\mathrm{UE}_{1}\right) \mathrm{B}} \& \& P L_{D 2 D}<=P L_{h d 2 d\left(U E_{2}\right)}$ and $\operatorname{SINR}_{h}<=\operatorname{SINR}_{\text {threshold } \& \&} \operatorname{SINR}_{d 2 d\left(U E_{2}\right)}<=\operatorname{SINR}_{\text {threshold }}$.

4. Set $x_{d^{*} h}=1$, Removal of Resources taking Complete D2D Users $D=D-\left\{d^{*}\right\}$; otherwise go to step 2.

The sum throughput of whole scheme is the sum of two equations (7) and (12).

\section{Evaluation and Simulation}

\subsection{Simulation Parameters}

We perform general simulations to evaluate the efficiency and throughput of proposed methods. Each user calculates SINR from the received signal power and interference power level, and then feeds back the channel quality indicator (CQI) to base station (BS). Table I summarizes a list of simulation parameters and their default values. Link variation and various modulation and coding schemes are performed based on the CQI at the BS. The subcarrier and bit allocation signal are transmitted by control channel to user. For HUE, two different scheduling algorithms are employed: Round robin (RR), comparative Fair (CF). The transmitter power of HUE is controlled using the same fixed SNR target in a limitation $(\mathrm{SNR}=13 \mathrm{~dB})$. The transmitters of $\mathrm{D} 2 \mathrm{D}$ pair's use the same fixed transmit power $\left(P_{d}=0.05 w\right)$.

Table 1. Simulation Parameters and Values

\begin{tabular}{|l|l|}
\hline \multicolumn{1}{|c|}{ Parameter } & \multicolumn{1}{c|}{ Values } \\
\hline Cell Layout & Hexagonal Network \\
\hline Spectrum allocation & $20 \mathrm{MHz}$ \\
\hline HUE distribution & uniformly \\
\hline Carrier Frequency & $2 \mathrm{GHz}$ \\
\hline Radius (Inter site distance) & $500 \mathrm{~m}$ \\
\hline RB Bandwidth & $180 \mathrm{KHz}$ \\
\hline Base station Tx power & $20 \mathrm{w}$ \\
\hline Base station height & $30 \mathrm{~m}$ \\
\hline Number of subcarrier per RB & 100 \\
\hline Shadowing fading & $8 \mathrm{~dB}$ \\
\hline UE noise range & $9 \mathrm{~dB}$ \\
\hline
\end{tabular}

\subsection{Results Discussion}

In this part we evaluate and discuss the simulation results.

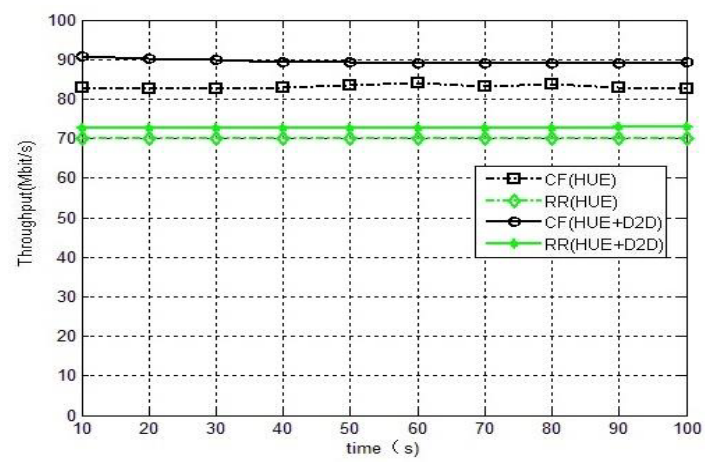

Figure 3.Scheme throughput for different scheduling algorithms with the proposed acquisitive resource block reuse algorithm.

Scheme throughput change with the time for different scheduling algorithms (RR, CF) in case of scenario 1 and 2 as shown in Fig. 3. Scenario 1 is a pure HetNets with $\mathrm{H}=40$. Scenario 2 is a mixed network with $\mathrm{H}=26, \mathrm{D}=4$ four D2D pair. For both cases, the RR performance is poor because it never considers the current channel condition and always allocates the same resources to all users. The CF performance is good because CF not only does consider the channel quality indicator (CQI) but also allocates resources in a fair proportion. In addition, for both (RR, CF) scheduling algorithms, the scheme throughput is higher when D2D communication reuse the RBs of HetNets. 
Scenario 1: HUE with set of $\mathrm{H}=40$.

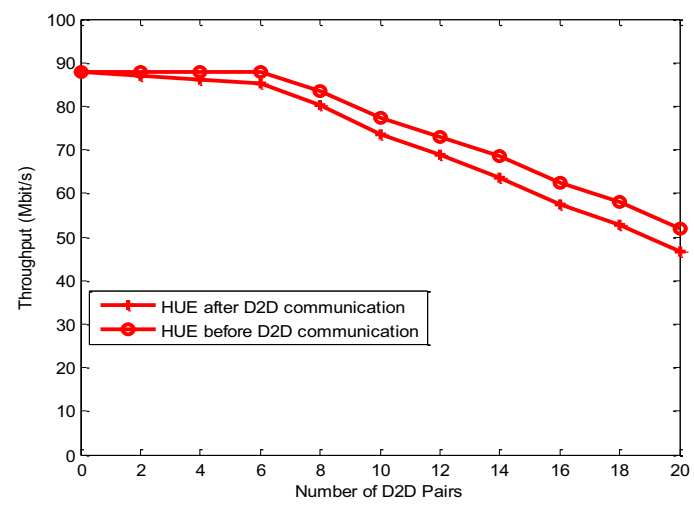

Figure 4.1 Scheme throughput comparison in HUE before and after D2D mode (Scenario D2D pair $=0: 2: 20$ )

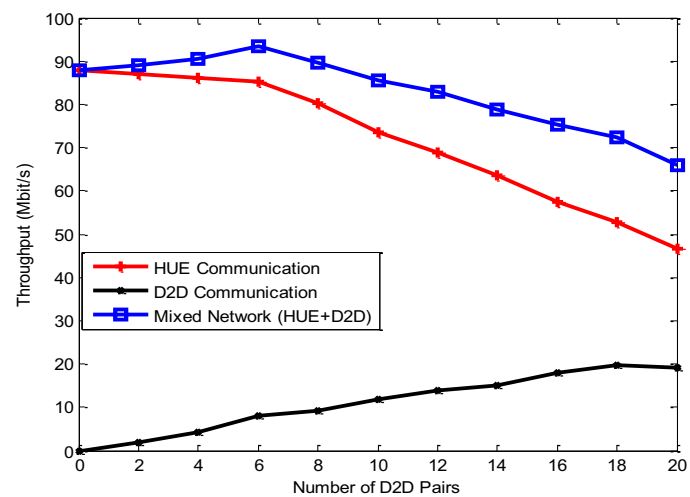

Figure 4.2 Throughput factor in HUE, D2D and mixed network mode (Scenario D2D pair $=0: 2: 20$ )

Scheme throughput in both HetNets and the mixed network, when the total number of links in the HUE is fixed $\mathrm{N}=40$. The evaluation of throughput in HUEs before and after D2D communication is shown in Fig. 4.1.The HetNets throughput is lower after D2D communication than before, because of the interference from the D2D connections. Scheme throughput factor in HUE, D2D and mixed network which clearly explains that mixed network throughput is higher as compared to HetNets and D2D mode shows in Fig. 4.2.

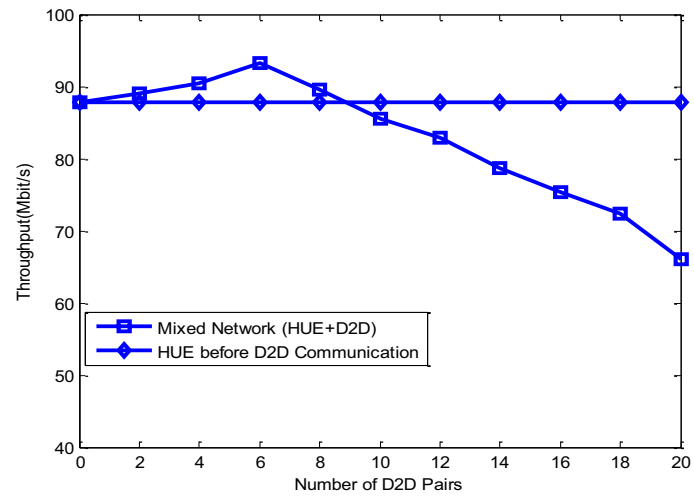

Figure 4.3 Scheme throughput comparisons in HUE before D2D and mixed network communication (Scenario D2D pair $=0: 2: 20)$.

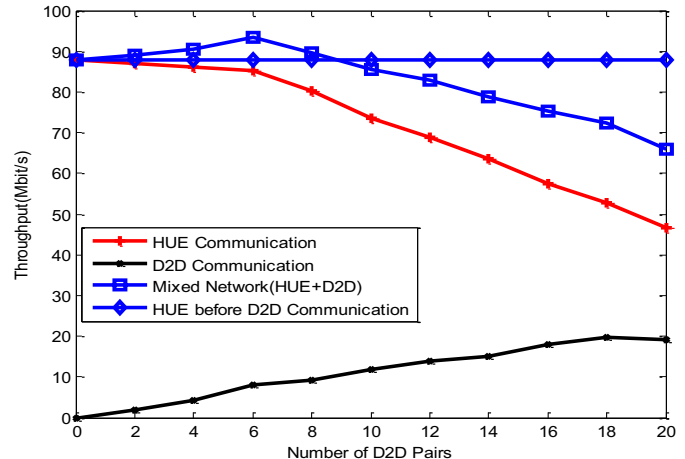

Figure 4.4 Scheme throughput comparison between HUE, D2D, mixed network and HUE before D2D communication (Scenario $\mathrm{D} 2 \mathrm{D}$ pair $=0: 2: 20)$

Component of the scheme throughput in HUE before D2D and mixed network communication are shown in Fig. 4.3. Comparison of system throughput between pure HetNets and mixed network are shown in Fig. 4.4. When, the scheme is pure HetNets. After D2D links, the scheme throughput is increased. Due to the fixed total links in the HUE, the scheme throughput cannot increase considerably with the $D$ increase as the $\mathrm{H}$ decrease. Results show clearly that when the primary service reaches the upper limit, the system throughput has increased.

Scenario 2: Mixed network HUE and D2D users with set of $\mathrm{H}=26, \mathrm{D}=4$.

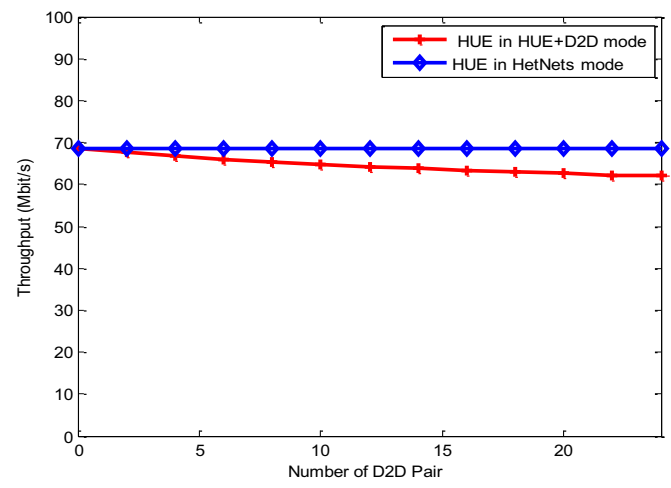

Figure 5.1Comparison of throughput in HUE before and after D2D form

(Scenario D2D pair=0:2:24).

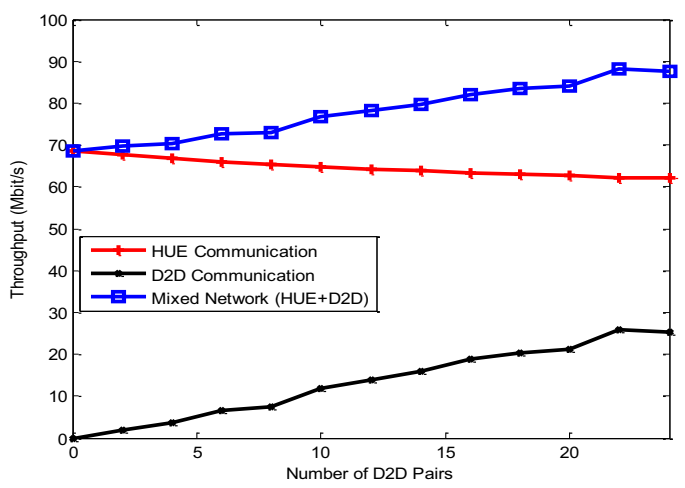

Figure 5.2 Throughput component in HUE, D2D and mixed network form(Scenario D2D pair=0:2:24).

System throughput both in the HetNets and the mixed network when the number of HUEs in the HetNets is 
fixed. The comparison of throughput from HUE between pure HetNets mode and mixed mode is shown in Fig 5.1. Because the number of HUE is fixed to 26 , the throughput in pure HetNets is a straight line. The HetNets throughput is worse in mixed mode, which is due to the interference from the D2D network. Component of the scheme throughput in D2D, HetNets and mixed network which clearly describe that mixed network throughput is higher as compared to D2D and HetNets network shows in Fig 5.2.

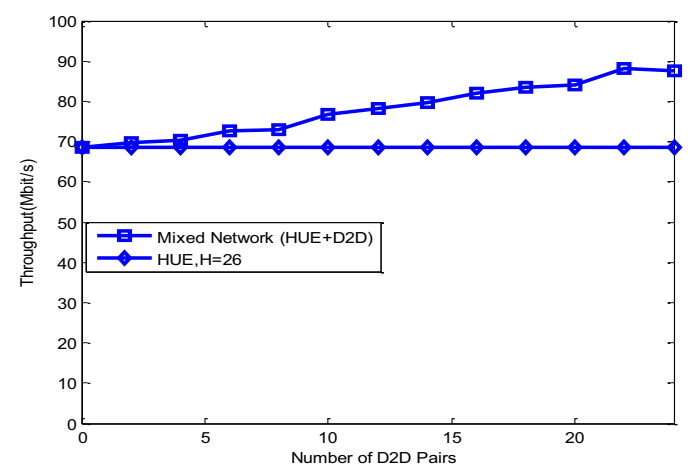

Figure 5.3Scheme throughput comparisons in HUE and mixed network communication (Scenario pair $=0: 2: 24$ ).

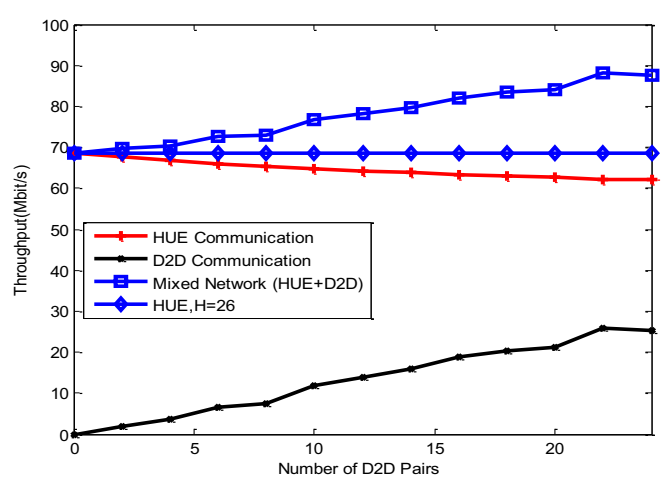

Figure5.4 Scheme throughput comparison between HUE, D2D, mixed network and HUE before D2D communication

(Scenario pair $=0: 2: 24$ ).

Component of the scheme throughput in mixed network and HetNets shows in Fig. 5.3.The comparison of scheme throughput between pure HetNets, D2D and mixed network shows in Fig. 5.4, when the scheme is pure HetNets. After D2D communication towards the HetNets, the scheme throughput increase for a long range. Due to the increasing number of D2D links in the HUE, the scheme throughput increase extensively. We can find out that the D2D links are not up to an upper limit.

\section{Conclusion}

D2D is an advanced technology that can increase the spectral efficiency and throughput by reusing the resource of HUE. This technology offers a beneficial complement to organization approach. The network performance improves when D2D communications share resources with the primary network. But, D2D transmitter causes interference to the UE receiver during the D2D LTE downlink HetNets period. In this article we have investigated how to reduce the effects of interference between D2D and HUE, in order to improve the system sum throughput for D2D.We have proposed a combined resource allocation and resource reuse method with objective of mitigation the interference between D2D and HUE. Proposed method ensures the D2D mode selection is a realistic way and mitigates the interference. We formulated that the system performance improves through calculation of the sum throughput in the scheme before and after D2D communication in LTE HetNets. Simulation results show that efficiency and throughput have been improved for the proposed approach

\section{Acknowledgment}

This paper is funded by the International Exchange Program of Harbin Engineering University for innovation-oriented Talents Cultivation, the National Natural Science Foundation of China (Grant NO. 61301095, NO. 51374099), the Natural Science Foundation of Heilongjiang Province, China (Grant No. F201345) and the Fundamental Research Funds for the Central Universities of Ministry of Education of China (Grant No. HEUCF150812).

\section{References}

1] Z. Liu, T. Peng, S. Xiang, and W. Wang, "Mode selection for Device-To-Device (D2D) communication under LTE-Advanced network," Proc. 2012 IEEE International Conference on Communication (ICC), 5563-5567, (2012).

[2] K. Doppler, M. Rinne, C. Wijting, C. B. Ribeiro, and K. Hugl, "Device-to-Device communication as an underlay to LTE-advanced networks," IEEE Commun. Mag., 47(12), 42-49, (2009).

[3] C. H. Yu, O. Tirkkonen, K. Doppler, and C. Ribeiro, "On the performance of Device-to-Device underlay communication with simple power control," Proc. IEEE Vehicular Technology Conference, 1-5, (2009).

[4] P. Janis, V. Koivunen, C. Ribeiro, J. Korhonen, K. Doppler, and K. Hugl, "Interference-aware resource allocation for Device-to-Device radio underlaying cellular networks," Proc. IEEE $69^{\text {th }}$ Vehicular Technologies Conference, 1-5, (2009).

[5] C. H. Yu, O. Tirkkonen, K. Doppler, and C. Ribeiro, "Power optimization of Device-to-Device communication underlaying cellular communication," Proc. IEEE International Conference on Communication, 1-5, (2009).

[6] K. Doppler, C. H. Yu, C. Ribeiro, and P. Janis, "Mode selection for Device-to-Device communication underlaying an LTE-Advanced network," Proc. IEEE Wireless Communications and Networking Conference (WCNC), 1-6, (2010).

[7] T. Peng, Q. Lu, H. Wang, S. Xu, and W. Wang, "Interference avoidance mechanisms in the hybrid cellular and Device-to-Device systems," Proc. IEEE 20th International Symp. on, Personal, Indoor and Mobile Radio Communications, 617-621, (2009). 
[8] T. Chen, G. Charbit, and S. Hakola, "Time hopping for D2D communication in LTE cellular system," Proc. IEEE Wireless Communications and Networking Conference (WCNC), 1-6, (2010).

[9] G. Yu, Z. Zhang, Y. Chen, P. Cheng, and P. Qiu, "Subcarrier and Bit allocation for OFDMA systems with proportional fairness," Proc. IEEE Wireless Communications and Networking Conference (WCNC), 1717-1722, (2006).

[10] M. Zulhasnine, C. Huang, and A. Srinivasan, "Efficient resource allocation for Device-to-Device communication underlaying LTE network," Proc. IEEE 6th International Conference on Wireless and Mobile Computing, Networking and Communications, 368-375, (2010).

[11]F. Graziosi and F. Santucci, "A general correlation model for shadow fading in mobile radio systems," IEEE Commun. Lett. 6(3), 102-104, (2002). 